\title{
Gestión del análisis de peligros y puntos críticos de control
}

\section{Hazard-analysis and critical-control-points management}

\section{María Juliana Moreno Guavita}

Ingeniera química, estudiante de maestría en Ingeniería Industrial. Docente de la Fundación Universitaria Cedinpro. Bogotá, Colombia.

Contacto:Ing.juliana@yahoo.com.co

Fecha de recepción: 10 de noviembre de 2011

Fecha de aceptación: 17 de abril de 2012

Clasificación del artículo: Revisión

Palabras clave: cambio organizacional, control de alimentos, industria alimentaria.

Key words: organizational change, food control, food industry.

\section{RESUMEN}

El propósito de este artículo es presentar una revisión teórica de los beneficios y limitaciones del sistema "Análisis de riesgos y puntos críticos de control o HACCP (Hazard Analysis Critical Control Points)". Se ha demostrado que la implementación del sistema HACCP es una buena elección como instrumento regulador y preventivo en las industrias alimenticias, pero no es suficiente, tiene limitaciones como: altos costos de infraestructura, falta de concientización, tiempo de ejecución, actividades de supervisión y documentación excesiva, entre otras. Quizás sea porque la industria es dinámica, evolutiva, flexible, rápida y no está limitada.

\section{ABSTRACT}

The purpose of this article is to present a theoretical review of the benefits and limitations of the system called HACCP (Hazard Analysis Critical Control Points). The HACCP system has proved to be a good implementation choice (with its seven principles) as a regulatory and preventive instrument within the food industry. However, HACCP is not enough due to some limitations, namely its high infrastructure costs, the lack of widespread awareness, its implementation time, the demanding monitoring and excessive documentation, among others. This might occur since industry, in general, is dynamic, evolutionary, flexible, fast and unlimited. 


\section{INTRODUCCIÓN}

El Sistema de análisis de peligros y puntos críticos de control, bien conocido por sus siglas como HACCP, "tiene un enfoque científico para tratar el control del proceso, diseñado para prevenir y asegurar la aplicación de controles en cualquier punto de un sistema de producción de alimentos" [1] es preventivo, sistemático y lineal, está basado en siete principios que son: "análisis de riesgos en la planta de producción, decidir sobre los puntos críticos de control (PCC), determinar los límites críticos, establecer procedimientos para vigilar los PCC, establecer medidas correctivas, procedimientos de verificación y crear un sistema de registro"[2]. Con el objetivo de buscar y garantizar la seguridad de los productos alimenticios y contribuyendo al aseguramiento de la inocuidad que es "la gestión operativa de seguridad alimentaria, que se obtienen por las diferentes cadenas de alimentos" [3]. Este sistema es avalado y autorizado por diferentes organizaciones internacionales como la Organización Mundial de la Salud o CODEX Alimentarus, la Organización Mundial del Comercio o OMC, la Organización Mundial de Salud Animal o OIE y la Convención Internacional para la Protección de los Vegetales o CIPV, entre otras.

HACCP y otros sistemas de calidad deben ser implementados en las empresas para garantizar la calidad de sus productos y servicios, teniendo en cuenta que calidad "Es calidad de trabajo, calidad del servicio, calidad de información, calidad de proceso, calidad de la gente, calidad del sistema, calidad de la compañía, calidad de objetivos," [4], con lo cual, los gerentes deben gestionar su proceso de implementación para evidenciar sus grandes resultados. Dichos resultados, que han obtenido algunas compañías, son los que se pretenden dar a conocer, evidenciando el alcance de la gestión, consultando y detectando sus grandes beneficios y, a la vez, identificando sus barreras o limitantes.

Para tal fin se abordará el tema de la siguiente manera: inicialmente se comenta el método de localización, selección y evaluación de algunos resultados que se han obtenido de algunas implementaciones, se evidencian dichos resultados, se discuten, se analizan y finalmente se realizan conclusiones.

\section{MÉTOdO DE LOCALIZACIÓN, SELEC- CIÓN Y EVALUACIÓN DE LA INFOR- MACIÓN}

El sistema de HACCP nace de la preocupación de los consumidores por adquirir alimentos inocuos, lo que les exige, a los responsables del tema, considerar algunas normas de carácter, nutricional, higiénico, sanitario y ambiental, contribuyendo a introducir conceptos de calidad que satisfagan las expectativas de los clientes [5]. HACCP fue una herramienta de gestión desarrollada en la década de 1960 para garantizar la seguridad de los alimentos en los vuelos espaciales, pero hasta el año 1986, la Academia Nacional de la Ciencias apoya y promulga la implementación de HACCP. Desde esta fecha el sistema se adapta bajo organismos mundiales como la Organización de Agricultura y de Alimentos y La Organización Mundial de la Salud CODEX Alimentarus [6]. Con lo cual, en el 1986 nacen los criterios, bajo las cuales se rige el sistema, se precisan conceptos, características, métodos y principios para la gestión de la inocuidad de los alimentos.

Como se mencionó, desde el año 1960 hasta la actualidad, existen datos, avances e información sobre el sistema HACCP, estos guían e intentan 
integrar los procesos para planificar, captar, dinamizar, organizar y administrar recursos y talentos, dentro de los límites de tiempo y costos, según el propósito de cada organización.

Lo más interesante de este artículo es analizar los resultados que se obtienen tras dicha gestión en algunas compañías a nivel mundial, por lo tanto, el método de localización de la información tiene asiento en los últimos años, pues esta gestión, por proceso del HACCP, toma usualmente no menos de tres a cinco años [7].

Se ajustan a esta investigación compañías que hayan aplicado al plan por proceso de HACCP, adaptables a cualquier tipo de industria alimenticia, siendo estos los criterios de selección. Se proceden a la evaluación de la información, donde el criterio de apreciación de HACCP lo dan los resultados tras su implementación.

La localización de la información útil para este artículo se consultó en una base de datos donde se destacan revistas indexadas en Ebson, Elsevier, Dialnet, Journal, entre otras. Esta consulta se realiza con una preselección de artículos científicos, se consulta tesis doctorales y disertaciones en bibliotecas virtuales.

\section{IMPLEMENTACIONES DEL SISTEMA HACCP}

El término inocuidad es necesario, primordial e implícito para la salud de los mismos actores y personas ajenas a la manipulación de los alimentos, es un término de alto interés en la sociedad actual. Si no fuera por esto, los brotes de intoxicación "que resultar de la ingestión de alimentos que contienen niveles inusualmente altos de histamina" [8] irían cada vez en aumento; estudios en Inglaterra entre los años 1992 y 1996 analizaron brotes de enfermedades transmitidas por los alimentos, específicamente la Salmonella (un género de gram negativa, bacilos anaerobios facultativos, generalmente móviles pertenecientes a la familia Enterobacteriaceae) [9], proporcionando información sobre agentes etiológicos, vehículos y factores que contribuyeron a dichos brotes, las cuales arrojaron un cifra de 135 brotes en diferentes alimentos [10]. Igualmente, autores como Smerdon, Adak, Brien, Gillespie en el año 2001, en la división de enfermedades gastrointestinales en Londres, lograron información sobre grandes brotes que se habían producido por enfermedades infecciosas intestinales en el Reino Unido, como resultado del consumo de carnes que no fueron manipuladas adecuadamente [11].

El comité de expertos de la Organización de las Naciones Unidas para la Alimentación y la Agricultura, FAO, sobre la seguridad alimentaria, hace hincapié en que los alimentos contaminados son probablemente el problema de salud más extendido en el mundo y son una causa importante para la baja productividad económica de este sector, por lo tanto, declara la necesidad de gestionar un proceso que prevenga la infección de los alimentos antes, durante y después de su manejo y no solamente como un control final después de su manipulación. Agrega, que hay necesidad de aplicar un proceso de gestión, HACCP; que ha sido descrito, como el medio más viable para prevenir enfermedades transmitidas por alimentos [12].

En teoría, su aplicación ofrece ventajas significativas como la inocuidad de alimentos, facilita la inspección por parte de las autoridades respecto a la reglamentación, promueve el comercio internacional, aumenta la confianza en los consumidores y posee compatibilidad con la aplicación del sistema de gestión de calidad ISO 9000 (la serie de certificación ISO 9000 para las empresas de alimentos incorpora los siete principios de HAC$\mathrm{CP}$ y deben ser aplicables a todos los aspectos relacionados con la calidad de los alimentos) [13]. La gestión por proceso del HACCP es un medio eficaz y racional que garantiza la seguridad ali- 
mentaria desde el inicio de su producción hasta su consumo, utilizando una metodología de "granja hasta la mesa" [14]. Lo que reduce los costos de producción por concepto de inspección y decomiso de productos contaminados o deteriorados, y el consiguiente impacto económico, político y social [15].

Se puede decir que HACCP es teóricamente viable y confiable, ahora se indagara si, en la práctica, es igualmente viable, cuáles son sus beneficios y limitaciones.

\section{Puesta en marcha del sistema HACCP.}

Diferentes países han desarrollado estrategias para la implementación de HACCP, desde el año 1998 el incremento en la aplicación a marcado la pauta, se cifra en un $100 \%$ para las grandes empresas, un $70 \%$ para las medianas y un $40 \%$ para las pequeñas, en países pioneros como Italia, Australia y Tailandia; en otros países en progreso ha tenido un avance dependiendo de su nivel de desarrollo, exportaciones, importaciones, consumidores y alianzas entre el gobierno y empresarios [16].

En el año 2007, en Turquía, donde antes de la aplicación del sistema HACCP, existían varios circunstancias que dejaban expuestos los alimentos a la contaminación, como: la producción en volumen (producir cantidad y no calidad), aparición de cadenas de comida cada vez más extensas y más complejas, el no posicionamiento de marca, la no especialidad alimentaria, aumento de consumo de comida rápida, vendedores ambulantes y crecimiento del comercio internacional sin control; su solución inmediata era la implementación de la gestión por proceso del HACCP, la cual fue aceptada por un grupo de empresas, donde, después de gestionar su proceso, sus resultados arrojaron que, en aproximadamente el $91.3 \%$, se identificaron mejoras respecto a la inocuidad de los alimen- tos y un aumento significativo en la confidencialidad del cliente con el producto [17]; sin embargo, se encontraron algunos limitantes como: la falta de programas previos con un $92,2 \%$ (barrera clave para todas las empresas investigadas); falta de personal capacitado con el $91.3 \%$; falta de conocimiento general sobre el sistema HACCP, que fue de $83,5 \%$; falta de tiempo de $88,7 \%$; falta de motivación de los empleados $83,5 \%$ y terminología complicada $87,0 \%$. Al ser aplicado, HACCP tiene ventajas y desventajas, estas últimas radican en no tener capacitación y conciencia preventiva. Resultados similares se obtiene Hielm y sus colaboradores, donde realizaron encuesta a 87 empresas de fabricación de alimentos para distribuirse a 870 empleados, preguntando sobre la estrategias de gestión de higiene de alimentos, sus resultados fueron positivos acerca de la calidad y seguridad de los productos; además, se le preguntó qué les había causado mayores dificultades en HACCP y las respuestas fueron: elegir los puntos críticos de control y la documentación [18].

Sean beneficios o limitantes, es claro que para toda industria alimenticia es necesario el HACCP, aunque no todas las empresas lo vean como una necesidad si no como un incentivo económico, por ejemplo, un caso en Turquía de la industria de manipulación de pavo (alimento de alto costo) aplicó HACCP, antes de su aplicación el 49,9\% de la producción se perdía por contaminación de Salmonella y otros agentes microbianos, después de su aplicación esta pérdida se redujo al 26,6\%, brindando seguridad al consumidor y progreso monetario a la empresa [19].

Investigaciones realizadas por Shaosheng y Jiehong en el año 2007 basadas en una encuesta de 117 empresas de alimentos en la provincia de Zhejiang, China, presenta los resultados con respecto a la adopción del sistema de gestión HACCP, los hallazgos sugieren y aportan la siguiente comparación: las empresas que no han adoptado un sistema HACCP, en cotejo con empresas que sí lo han 
adoptado, pueden ser identificadas por los siguientes criterios: son pequeñas y medianas empresas (observaciones anteriores han sugerido que grandes plantas encontrarían la aplicación de HACCP mucho más fácil que las plantas más pequeñas [20]), proveedoras del mercado nacional, no han aplicado otros sistemas de gestión de calidad y sus administradores o gerentes poseen niveles relativamente bajos de educación; dando así una percepción limitada al sistema HACCP. Adicionalmente, sus autores afirman dos supuestos respecto a la introducción al sistema HACCP en las PYMES: afirman que es una gran carga por tener un alto costo de implementación y necesita de sistemas de regulación estricta; asimismo, hacen hincapié en que, una vez que el público sea consciente de la eficacia del sistema HACCP para asegurar la inocuidad alimentaria, es probable que los clientes prefieran alimentos producidos por empresas que emplean un sistema HACCP, por tanto, está a la voluntad de las empresas adoptar el sistema HACCP [21].

En lo que respecta a Colombia, en el año 2003 se fomentó la asesoría y la formación del HACCP donde, el Servicio Nacional de Aprendizaje, SENA, la Universidad Nacional de Colombia, la Corporación Colombiana Internacional y la Corporación Colombiana de Investigación Agropecuaria, trabajaron en un proyecto titulado "Programa integral de transferencia de tecnología para la producción limpia y la comercialización de hortalizas en la Sabana de Bogotá", donde se desarrolló fuertemente un componente de calidad y de "buenas prácticas para la Agroindustria BPA", para inculcar las buenas prácticas en la manipulación de alimentos, desde la agricultura hasta su consumo, apoyados por el sector comercio sólo en la parte de asesoría y capacitación [22]. Es relevante mencionar que sectores de la educación tienen el desafío de enseñar las mejores prácticas en manipulación de alimentos y concientizar que este proceso es una excelente estrategia de negocio para el nuevo enfoque en la seguridad e inocuidad de alimentos.
Adicionalmente, en Colombia, en julio de 2005 se aprobó la norma técnica NTC 5400 sobre las Buenas Prácticas Agrícolas para Frutas, hierbas aromáticas, hierbas culinarias y hortalizas. Adicionalmente, el Instituto Colombiano de Normas Técnicas y Certificación, ICONTEC, da a conocer los requisitos generales sobre la salubridad e inocuidad de los alimentos para las organizaciones que deseen aplicarlo [22].

HACCP se implementó en el proceso de germinación de semillas en el Valle del Cauca Colombia en el año 2009, identificando sus PCC, entre ellos: recepción de semillas, eliminación de impurezas, suspensión de germinación (escaldado de las semillas germinadas), empaque, almacenamiento, tabla del análisis de riegos y la tabla de control; se establecieron límites críticos, se propusieron medidas preventivas, acciones correctivas y procedimientos para obtener semillas totalmente inocuas [23]. La implementación del sistema HACCP beneficia la producción agroalimentaria, aumenta la aceptabilidad internacional e incrementa los ingresos en divisas procedentes por el comercio.

Una investigación que se realizó en el departamento de alimentos y ciencias nutricionales en Emiratos Árabes, por D. Al-Kandari y D. Jukes en el año 2011, destacaron términos como eficacia y eficiencia, donde hacen una constante presión para encontrar mecanismos más eficientes y más eficaces para llevar a cabo el control de los alimentos; lo que ha llevado a la población en Emiratos Árabes a reconocer la importancia internacional que tiene el sistema, identificando que HACCP es una herramienta de orientación y de control que repercute de manera importante sobre el papel de los organismos gubernamentales. Se identificaron dos elementos claves para el fortalecimiento de la implementación: el primero es la posición interna del gobierno, que tiene su ventaja en el compromiso, flujo de efectivo y liderazgo; y el segundo el comercio, pues el sistema abre 
oportunidades externas, es decir, comercializar fuera del país. Dicha investigación concluye que el HACCP tiene un reconocimiento internacional, no tiene amenaza donde el gobierno no pueda enfrentarla y es buena estrategia para facilitar y fomentar la atención comercial [24].

Ajena a esta investigación, el HACCP fue implementado en una planta de producción de "Kenkey" maíz fermentado, en Accra África; esta investigación se ejecutó posterior a la implementación de buenas prácticas de manufactura o BPM que es un "conjunto de principios y recomendaciones técnicas que se aplican en el procesamiento de alimentos para garantizar su inocuidad y su aptitud para evitar su adulteración" [25]; HACCP se ejecutó desde el inicio de la producción, comenzando con la supervisión de materias primas, aditivos, elaboración, procesamiento y con el análisis de factores de riesgo, ajustando éstos a los límites críticos que garantizan la seguridad del maíz fermentado. Se realizaron análisis de laboratorios, obteniendo que el nivel de aflatoxinas, toxinas totales en el maíz, en las muestras de "Kenkey", antes de la implementación de BPM y de HACCP, eran entre el rango de 64,1- 19,6 g toxina/kg de Kenkey y después de la implementación de HACCP el nivel estaba entre 17,2 - 14,5 $\mathrm{g}$ toxinas $/ \mathrm{kg}$ de Kenkey. Acertaron que la implementación de los sistemas de BPM y de HACCP son efectivos y mutuamente complementarios; impidiendo la proliferación de materia bacteriana y dando un entorno libre de microorganismo, manteniendo así el control en la calidad de los alimentos. Adicionalmente, esta investigación hace un llamado a seguir trabajando y mejorando la gestión por proceso del HACCP, de tal manera que sus resultados sean excelentes y se tome conciencia de su aplicación y necesidad [26].

Al seguir indagando sobre la aplicación de HAC$\mathrm{CP}$ en un caso especifico, como en productos cárnicos, la investigación realizada por E.S. Maldonado, S.J. Henson, J.A. Caswell, L.A. Leos,
P.A. Martinez y G. Aranda, J.A. Cadena, en México en abril del 2005, analiza los costos de implementar HACCP y los beneficios de su gestión. Inicialmente, realizaron un estudio alrededor de 160 empresas con respecto al interés de implementar el sistema, con una tasa de respuesta de solo el $58 \%$, donde el $18 \%$ de las empresas aprobó totalmente HACCP, el 20\% no tenía interés en adoptarla y el resto desertó en la aplicación; algunos resultados fueron: su principal beneficio es la reducción microbiana invirtiendo en equipos, en costos operacionales y en pruebas microbiológicas (que representa la mayor parte de la aplicación); la capacitación y la formación del personal es un problema altamente significativo [27]. De igual manera, HACCP fue implementado en la fábrica de productos lácteos COVALACT S.A en Rumania, allí se implementó el sistema, hallando cuatro puntos críticos de control, tres puntos biológicos y uno químico, asegurando un buen análisis y los limites de los PCC por medio de tecnología dispendiosa, eliminando los riesgos asociados a la fabricación de este producto y obteniendo un producto con cero denuncias vinculada a su fabricación, depósito y comercialización [28].

Dichas barreras deben ser vencidas y los beneficios deben ser acentuados para ver en HACCP una alternativa de solución, una meta al perfeccionamiento de la seguridad alimentaria, no verlo como una opción o requisito si no como una necesidad. En Estados Unidos, en el año 2000, se realizó una investigación que estableció, como política legal, la reducción del patógeno Salmonella en cadáveres de animales para su posterior consumo. En varias organizaciones se implementó este proceso basados en tres etapas fundamentales: 1) Buen establecimiento del habitad de animales, 2) excelente establecimiento de sacrificio y 3) excelente procesamiento del producto de carne y aves de corral. Con estos requisitos establecidos, los resultados obtenidos tras la manipulación de carne fueron exitosos, se disminuyó totalmente el nivel de bacterias toxicas y hubo mejora en el 
método de aplicación de HACCP por procesos [29]. Pese a no ser evaluada como valor legal si no como valor social.

Sistemas HACCP también son utilizado en la industria láctea en los Estados Unidos, a mediados del año 1980, donde la Asociación Internacional de alimentos lácteos, apoyada en el Distrito Federal Washington, D.C. y otras organizaciones, popularizaron el uso de HACCP. Sin embargo, en este tiempo, el sistema HACCP no era realmente efectivo debido a su gran infraestructura, tanto así, que cinco grandes empresas del momento o “controladores" como lo llama Allen R. Sayler en su investigación fueron las que originaron la evolución, mejora y el perfeccionamiento de los programas de HACCP en la industria láctea [30]. No obstante, en el año 2002 "La asociación Internacional de Alimentos Lácteos o IDFA concreta el HACCP para el manual de lácteos (última edición 2010), donde proporcionan 21 modelos para los diferentes productos lácteos y amplias recomendaciones sobre los programas funcionales para la plena eficiencia del HACCP.

Otra aplicación de HACCP es en la producción de aguardiente y de Ouzo, licor de origen griego formado por etanol, derivados de la uva, caña de azúcar, anís, hierbas y semillas" [31], informa sobre los riesgos o los puntos críticos a controlar en este proceso, mostrando que los riesgos fueron analizados y eficientemente controlados, aplicando HACCP, evidenciando que este sistema es seguro y controla la contaminación del producto. Paralelamente, sus autores aseveran que si el HACCP se logra vincular con el TQS "filosofía de calidad total en organizaciones de servicios" [32] se obtendrán mejores resultados respecto a la formación y perfeccionamiento del personal, perfilando la cooperación entre la administración, el personal, la adhesión y la lealtad a la gestión por proceso, que facilitan requisitos de auditorías y registros internos [33]. Que implícitamente son de gran ayuda a la hora de formalizar un sistema de gestión integral. En complemento, Mahmoud El-Hofi y su grupo de investigación aseguran que HACCP tiene consecuencias para los mercados nacionales, internacionales y para la serie ISO 9000, lo que permite pensar que la ISO 9000 es un paso intermediario en la aplicación de HACCP [34].

Se han llevado a cabo otras investigaciones que integran el cuidado de los alimentos, el servicio de salud y la nutrición, por ejemplo en Foggia, Italia, donde se analizaron algunas organizaciones que deseaban adoptar el sistema HACCP, evaluando su viabilidad con índices cualitativos. Los índices identificados de manera general fueron: la adhesión, la simplicidad, la especificidad, la integridad y la viabilidad del sistema, además se determinaron cuatro grupos de trabajo para su implementación: grupo 1, que trabaja con la ayuda de un equipo calificado en HACCP; grupo 2, trabaja con la ayuda de un grupo de personal pero sin capacitación oportuna; grupo 3, trabaja con la ayuda de un experto no calificado; grupo 4, trabaja sin personal calificado y sin grupo de apoyo. Sus análisis revelaron que el proceso de HAC$\mathrm{CP}$, con el personal capacitado y el conocimiento profundo en el sistema, se asocia un mejor nivel cualitativo de todos los indicadores evaluados con un puntaje bueno, a excepción del indicador de integridad que fue excelente y hubo una mejor aceptación por parte del personal, mayor facilidad en el proceso de implementación y se alcanzaron valores medio-altos respecto a la reducción de contaminantes biológicos y físicos [35].

Estudios sobre la valides de los elementos del sistema HACCP demuestran si este es un sistema útil como un elemento de verificación, validación y control de alimentos. Por ejemplo, el estudio de V. Scott, analiza si el plan de HACCP controla eficazmente los riesgos y si determina los límites críticos en forma considerada, este estudio hace hincapié en el término reevaluación, definida como un proceso donde se revisan todas las hi- 
pótesis y conclusiones del HACCP, considerando cuestionamientos como: ¿El sistema seguirá siendo válido?, ¿los planes siguen siendo suficientes tras la evolución de la industria que lo ha implementado?, aún sabiendo que la revalidación confirma los resultados anteriores del HACCP (especialmente si los datos anteriores fueron limitados) o evalúa la validez de parámetros menos conservadores [36]. Con esto se da la oportunidad de pensar que, en el área empresarial existen diversas, distintas y continuas operaciones que conllevan a cambios significativos, ya sea tras la evolución del sistema, tras la implementación de nuevos productos, cambios gerenciales, apertura de nuevos mercados, diversificación, entre otras. Características que disponen de poca o ninguna información, inciertas y no constantes ni lineales, es aquí donde el termino revalidación es necesario, es decir, hay que revalidar el sistema por proceso de HACCP para que sea más flexible y asequible a las industria en continuo cambio, V. Scott afirma que el modelo de aplicación de HACCP siempre está ligado al termino revalidación.

Estos son algunos ejemplos reales de empresas dedicadas al sector que han implementado o aplicado algunos requisitos del sistema HACCP. A continuación se hace una discusión de la recopilación de estas investigaciones.

\section{DISCUSIÓN}

Al implementar el sistema HACCP en la industria, se evidencia que, efectivamente, garantiza la seguridad de los alimentos, proporcionando un alto grado de calidad, eliminando los posibles riesgos biológicos, químicos y físicos y evitando así atentar contra la salud pública, sugiriendo la producción de alimentos seguros desde el cultivador hasta el consumidor, reduciendo en gran medida la perdida de productos, salarios de inspección, decomisos de productos y resección económica, política y social.
En todo sistema de gestión por proceso, su implementación representa beneficios y restrictivos, en esta fase del artículo ya son claros los beneficios de la implementación de HACCP; ahora, es pertinente mirar algunos limitantes, "como la capacitación, el uso predominante de la lengua inglesa, la no uniformidad en manuales, la escasez de información técnica, muy pocas personas experimentadas en la industria y en algunas autoridades reguladoras y la compleja naturaleza de algunos productos" [37].

Una de las más importantes limitantes es el alto costo que conlleva su implementación "construcción o reconstrucción de la planta, higiene personal, equipos de limpieza o tecnología de saneamiento, pruebas piloto, pruebas microbacterianas y saneamiento de procedimientos, son los menos propensos a estar implementados en pequeñas empresas"[38], una camino viable es otorgar apoyo financiero por parte del gobierno a las organizaciones, especie de soporte o impuestos "preferenciales" para estimular la implementación del sistema HACCP, "el proyecto es viable sin embargo, reconoció que el apoyo y respaldo de la OMS, la FAO y los gobiernos nacionales eran un requisito previo esencial para su éxito" concluyó la cuarta reunión internacional del Foro de seguridad de alimentos de Noordwijk en marzo de 2001 [39]. En su investigación, Samira Sarter hace énfasis en que las instituciones pertinentes y el gobierno deben ser vitales en dicho proceso, hace referencia a cuatro principales responsabilidades: 1) política alimentaria, 2) evaluación de riesgos, 3) legislación y 4) autoridades públicas; la autora agrega itodos los gobiernos están enfocados en mejorar la inocuidad y salubridad de los alimentos?, ¿todos los gobiernos ven con la misma importancia los riesgos agroalimentarios? o más bien ¿todos tenemos conciencia de una política alimentaria? [40].

Otra desventaja del sistema es la identificación de los riesgos, debido a que todo el personal no tiene 
el mismo concepto de riesgo o la medida del riesgo o incluso no tienen claro ¿cómo se identifica un riesgo? o ¿cómo se mide el riego?; la solución que han acogido algunas industrias es la capacitación no solo en el tema de gestión por proceso del HACCP, si no, introducir temas como: tabulación de Cruz, información de vigilancia a agentes etiológicos, vehículos de agentes infecciosos, herramientas que facilitan el análisis de riesgos y, en algunos casos, introducen temas como la teoría del riesgo, entre otros. Por tanto, se requiere una evaluación y conocimiento de teorías del riesgo, de forma más amplia y eficaz cumpliendo los requerimientos del HACCP. "El sistema HACCP fue desarrollado inicialmente como una herramienta solo a nivel de gestión para procesos específicos y ahora es y se estableció para industrias de todo tipo de procesos y de conocimiento" [41].

La gestión, el talento humano, actitudes, rotación del personal y el compromiso hacia la organización, son una barrera que afecta el proceso de HACCP, "Una falta de comprensión de HACCP fue identificada como uno de los principales obstáculos para su aplicación, 63,5\% informó que realmente no sabían lo que era HACCP mientras que $23,5 \%$ informó que era demasiado complicada" [9]. Entre tanto, mientras exista una amplia gama de literatura sugiriendo la eficacia de HAC$\mathrm{CP}$ versus la mala conducta, la cortedad, la obligación, la exigencia, la ignorancia y, más aún, la falta de compromiso por parte del talento humano, muy seguramente se estará perdiendo tiempo y dinero que será irremplazable.

Este artículo apoya una de las conclusiones de la investigación por John Ehiri "el HACCP es un proceso de formación y de educación hacia los operadores de alimentos y hacia los funcionarios reguladores de alimentos, es y debe ser una condición que va mas allá de la realización y de los beneficios de una estrategia" [42]; agregando por Anita Eves "Hay pocas dudas de que HACCP tiene cada vez mayor aceptación en la industria de alimentos. Su puesta en práctica, sin embargo, requiere la comprensión de sus principios y su compromiso a través de todos los niveles en la fuerza de trabajo" [43]. La disminución de esta limitante es totalmente factible por medio de la capacitación del personal, como lo plantea Shaosheng Jin y sus colaboradores, que afirman "elevar el nivel de educación de los administradores, gerentes y colaboradores es una solución viable para aumentar la tasa de aplicación del sistema HACCP" [44]. Al enfrentan esta barrera el objetivo de HACCP será claro y preciso, dando una sociedad más consciente a su carestía.

Otra barrera identificada es no hacer posible que cada organización cumpla estricta, específica y conscientemente los principios planteados por HACCP, cada organización es única e irremplazable, no se debe pensar que todas las industrias deben cumplir con el proceso tal cual como está descrito (se tiene claro el objetivo pero no tiene que ser el mismo camino), es frecuente que las políticas (misión y visión) y las estrategias de cada industria sean únicas, cada quien define sus medidas de control y vigilancia (obviamente precisando los respectivos límites críticos), cada quien considera que es "termino critico indiscutiblemente en términos racionales", lo que lleva a pensar que las compañías ya no estarían de acuerdo con el sentido original de los principios de HACCP [45].

Se identificaron otros problemas tras la implementación del HACCP en la industrial de alimentos como: cuestiones relacionadas con el tiempo de ejecución, actividades de supervisión, costos de grabación y documentación excesiva [41].

Estas debilidades y las mencionadas anteriormente están citadas por los que vivieron o están experimentando la implementación de este sistema, estas industrias se ven obligadas a superar todas estas barreras, ya sea porque son totalmente conscientes de su importancia (que es lo fundamental 
y lo relevante) o por el cumplimiento de un requisito legal. Evidentemente, se reconoce que el sistema HACCP es necesario y funciona, pero no es lo suficiente para las industrias alimenticias, será ¿falta de más control?, ¿falta de más seguimiento organizacional y gubernamental?, ¿falta de más capacitaciones para concientizar a los trabajadores de las industrias alimenticias? o más aún ¿será que el sistema de gestión es el adecuado?

\section{CONCLUSIONES}

La industria alimentaria se enfrenta a enormes desafíos, como el esfuerzo por satisfacer las exigencias del consumidor, hacer que el producto sea más asequible, de mayor calidad, proporcionar la máxima seguridad posible y ser competitiva en el mercado. HACCP es reconocido internacionalmente como el mejor sistema de control para la producción de alimentos que existe en la actualidad [46]. Se fundamenta en siete principios donde el éxito de estos requerirá "en el compromiso y la cooperación plena de los empleados del sector alimentario" [47], inculca la identificación de riesgos, los analizan, toma decisiones bajo estos riesgos e identifica métodos para limitar la exposición de estos.

HACCP analiza el sistema de producción desde el inicio hasta el final, por ejemplo, un estudio realizado en Universidad de Bristol en el año 2009, basado en la exportación de leche de novillas tuvo que frenar el proceso de implementación, debido a que se detectaron varios y serios problemas en el manejo ambiental del hábitat de las novillas, de forma que se identificaron riesgos en la salud de las novillas que conlleva a un mala calidad del producto [48]. Se desertó de la implementación por no tener una infraestructura y tecnología en el hábitat de las novillas adecuada. Esta es la forma de operar del sistema, si se logra combatir estos aspectos y se tienen en cuenta otros factores como: métodos específi- cos de procesamiento, pruebas de materia prima, controles y evaluaciones por agentes externos; se tendría un mayor avance en la implementación, dando cumplimento paso a paso a cada uno de sus principios y manteniendo la inocuidad y sanidad de los alimentos.

Es muy frecuente que la industria evolucione, sea dinámica, flexibles, rápida y no esté limitada, cada organización debe ser consiente a dónde quiere llegar, pero independiente de cómo lo deben hacer, obviamente buscando siempre el beneficio común. "cada quien definen sus medidas de control y vigilancia según sus límites críticos, por tanto, ya no se estaría cumpliendo con el sentido original de los principios de HACCP" [45]. En la práctica, la aplicación consecuente de los siete principios es diluida y conduce a una disminución en la eficacia de los conceptos de seguridad de alimentos.

Se sabe que HACCP es una idea relevante como instrumento regulador, ha demostrado que su implementación es una buena elección, pero no basta sólo el HACCP, falta algo, quizás sea concientización, gestión autónoma o asequibilidad. "Sin embargo, HACCP ha alcanzado sus objetivos y seguirá proporcionando la base para la siguiente evolución de inspección de alimentos", "es una herramienta que ha hecho que inspectores, fabricantes y grupos de consumidores reflexionen acerca de la cadena de suministro de alimentos, evaluando sus puntos fuertes y debilidades que a veces son ocasionales, inherentes o inevitables" [49]. Por último, a menudo se dice que el sistema HACCP debe aceptarse como es, de hecho, el Dr. Buchanan (profesor y director del centro de seguridad alimentaria) afirma que "el sistema de HACCP es un medio eficaz para controlar problemas de seguridad alimentaria, donde cada vez, se está introduciendo en instalaciones de producción de alimentos" [50]. Sin embargo, el perfeccionamiento del sistema debe ser siempre un objetivo $\mathrm{y}$ tarea de todos. 


\section{REFERENCIAS}

[1] United States Department of Agriculture, "Modelo HACCP general para productos crudos, sin moler, de carne y aves", in Food Safety and Inspection Service, Septiembre, 1999.

[2] U.S. Department of Health and Human Services Food and Drug Administration Center for Food Safety and Applied Nutrition, Managing Food Safety, A Manual for the Voluntary Use of HACCP Principles for Operators of Food Service and Retail Establishments, April, 2006.

[3] L. G.M. Gorris, "Food safety objective: An integral part of food chain management", Food Control, Vol. 16, pp. 801-809, 2005.

[4] K. Ishikawa, ¿Qué es Control Total de la Calidad? El modelo japonés; Prentice Hall, 1985.

[5] Feigenbaum, Total quality control, 3ra ed, New York: McGraw-Hill, 1991.

[6] K. Ropkins y A. Beck, "Evaluation of worldwide approaches to the use of HACCP to control food safety", Trends in Food Science \& Technology, Vol. 11, pp. 10-21, 2000.

[7] S. Altekruse, D. Street, S. Fein and A. Levy, "Consumer knowledge of foodborne microbial hazards and food-handling practices", Journal of Food Protection, Vol. 59, No.3, March, pp. 287-94, 1996.

[8] S. Taylor, "Histamine food poisoning: toxicology and clinical aspects", Critical Reviews in Toxicology, Vol. 17, pp. 91-128, 1986.
[9] H. Ekperigin and K. Nagaraja, "Microbial food borne pathogens. Salmonella", Vet Clin North Am Food Anim Pract, Vol. 14, pp. 17-29, 2006.

[10] P. Panisello, R. Rooney, P. Quantick and R. Stanwell-Smith, "Application of foodborne disease outbreak data in the development and maintenance of HACCP systems", International Journal of Food Microbiology, Vol. 59, No. 3, pp. 221-234, September 2000 .

[11] W. Smerdon, G. Adak, S. O'Brien, I. Gillespie and M. Reacher, "General outbreaks of infectious intestinal disease linked with red meat, England and Wales", Journal commun dis public health, Vol. 4, No 4, pp. 259-267, 2001.

[12] Conferencia Regional FAO/OMS, Inocuidad de los Alimentos para las Américas y el Caribe San José, Garantizar la inocuidad de los alimentos en los ámbitos de la producción y la elaboración, Costa Rica, December 2005.

[13] J. Caswell y N. Hooker, "HACCP as an International Trade Standard", American Journal of Agricultural Economic association, Vol. 78, No. 3, pp. 775-779, Aug., 1996.

[14] W. Sperber, "HACCP does not work from Farm to Table", Food Control, Vol. 16, No. 6, July, pp. 511-514, 2005.

[15] Y. Pérez, Y.Suárez, J. Cura y A, Pérez, "Bases técnicas para la aplicación del sistema de análisis de peligro y puntos críticos de control (HACCP) desde la granja de pone- 
doras hasta la recepción y distribución de huevos para el consumo", Revista Electrónica de Veterinaria REDVET, Vol. 7, pp. 1-12, 2006.

[16] Países M. Lourdes, "El sistema de Análisis de peligros y de puntos críticos de control en la industria de alimentos", en FAO corporate document repository, Oficial Superior del Grupo de Enlace en Materia de Calidad de los Alimentos de la FAO, Agriculture and Consumer Protection, Vol. 28, pp. 26-34

[17] M. Baş, M. Yüksel and T. Çavuşoğlu, "Difficulties and barriers for the implementing of HACCP and food safety systems in food businesses in Turkey", Food Control, Vol. 18, No. 2, February, pp. 124130, 2007.

[18] S. Hielm, P. Tuominen, K. Aarnisalo, L. Raaska and R. Maijala, "Attitudes towards own-checking and HACCP plans among Finnish food industry employees", Food Control, Vol.17, pp. 402-407, 2006.

[19] E. Nganje, M Siaplay, S Kaitibie and T. Acquah, "Predicting Food Safety Losses in Turkey Processing and the Economic Incentives of Hazard Analysis and Critical Control Point (HACCP) Intervention", Agribusiness, Vol. 22, pp. 475-489, 2006.

[20] A. Alberini, E. Lichtenberg, D. Mancini and I. Galinato, "Was it something $i$ ate? Implementation of the FDA seafood HACCP program", American Agricultural Economics Association, Vol. 90, pp. 28-41, 2008.

[21] S. Jin, J. Zhou and J. Ye, "Adoption of HACCP system in the Chinese food industry: A comparative analysis", Food Control, Vol 19, No. 8, August, pp. 823-828, 2008.
[22] L. Castellanos, L. Villamil y J. Romero, "Incorporación del Sistema de Análisis de Peligros y Puntos Críticos de Control en la Legislación Alimentaria", Rev. Salud pública, Vol. 6, No. 3, pp. 289-301. 2004.

[23] D. Chaparro, Y. Portilla, A. Elizalde, N. vivas y C. Erazo, Estratégia ARPCC en la producción de semillas germinadas, $\mathrm{Fa}$ cultad de Ciencias Agropecuarias, Vol 7, 2009, pp. 36- 44.

[24] D. Al-Kandari and D. Jukes, "Incorporating HACCP into national food control systems Analyzing progress in the United Arab Emirates", Food Control, Vol. 22, No. 6, June, pp. 851-861, 2011.

[25] A. Díaz y R. Uría, Buenas Prácticas de Manufactura Una guía para pequeños y medianos agroempresarios, Instituto Interamericano de Cooperación para la Agricultura, IICA: Costa Rica, 2009.

[26] W. Amoa-Awua, P. Ngunjiri, J. Anlobe, K. Kpodo, M. Halm, A. Ewurafua, et al, "The effect of applying GMP and HAC$\mathrm{CP}$ to traditional food processing at a semi-commercial kenkey production plant in Ghana", Food Control, Vol. 18, No. 11, November, pp 1449-1457, 2007.

[27] E. Maldonado, S. Henson, J. Caswell, L. Leos, P. Martinez, G. Aranda, et al, "Cost-benefit analysis of HACCP implementation in the Mexican meat industry", Food Control, Vol. 16, No. 4, April, pp. 375-381, 2005.

[28] A. Madar and A. Neacsu, "The efficiency of applying HACCP system in milk Industrie. Case study. S.c.covalact s.a. for the Product "consumer milk 1.8\%", Annals of the University of Oradea", Economic Science Series, Vol. 17, pp. 942-954, 2008. 
[29] W. Schlosser, A. Hogue, E. Ebel, B. Rose, R. Umholtz, K. Ferris, et al, "Analysis of Salmonella serotypes from selected carcasses and raw ground products sampled prior to implementation of the Pathogen Reduction; Hazard Analysis and Critical Control Point Final Rule in the US", International Journal of Food Microbiology, Vol. 58, No. 1-2, pp. 107-111.

[30] A. Sayler, "Tools for a Functional Dairy HACCP Program", Dairy Foods, Vol. 10, December, 2011

[31] A. Varvagiannis, L. Margomenou and I. Zabetakis, OUZO and RAKI, Laboratory of Food Chemistry, Department of Chemistry, University of Athens, 15771 Panepistimioupolis: Athens, Greece.

[32] G.S. Sureshchandar, C Rajendran and R.N. Anantharaman, "A holistic model for total quality service", International Journal of Service Industry Management, Vol. 12, pp. $378-412,2001$.

[33] V. Scott, "How does industry validate elements of HACCP plans?", Food Control, Vol. 16, pp. 497-503, 2005.

[34] M. Efstratiadis and I. Arvanitoyannis, "Implementation of HACCP to large scale production line of Greek ouzo and brandy: a case study", Food Control, Vol. 11, No. 1, February, pp. 19-30, 2000.

[35] E. Mahmoud, E. El-Sayed and I. Azza, "Implementation of the hazard Mahmoud analysis critical control point (haccp) system to uf white cheese production line", Acta Sci. Pol., Technol. Aliment, Vol. 9, No. 3, March, pp. 331-342, 2010

[36] M. Panunzio, A. Antoniciello and A. Pisano, "System of Indexes and Indicators for the Quality Evaluation of HACCP Plans based on the Results of the Official Controls conducted by the Servizio di Igiene degli Alimenti della Nutrizione (Food and Nutrition Health Service) of the Local Health Authority of Foggia, Italy", Int. J. Environ. Res. Public Health, Vol. 4, No. 1, pp. 34-38, 2007.

[37] P. Jirathana, "Constraints experienced by developing countries in the development and application of HACCP", Vol. 9, pp. 97- 100, 1998.

[38] M. Conter, E. Zanar, S. Ghidini, L. Pennisi, A. Vergara, G. Campanini and A. Ianieri, "Survey on typology, PRPs and HACCP plan in dry fermented sausage sector of Northern Italy", Food Control, Vol. 18, pp. $650-655,2007$.

[39] A. Williams, A Smith, R Gaze, S. Mortimore, Y. Motarjemi and C.A Wallace, "An international future for standards of HACCP training”, Food Control, Vol. 14, pp. 111-121, 2003.

[40] S. Sarter, G. Sarter and P. Gilabert, "A Swot analysis of HACCP implementation in Madagascar", Food Control, Vol. 21, No. 3, March pp. 253-259, 2010.

[41] L. Unnevehr y H. Jensen, "The economic implications of using HACCP as a food safety regulatory standard", Food Policy, Vol. 24, No. 6, December, pp. 625-635, 1999.

[42] J. Ehiri, G. Morris and J. McEwen. "Implementation of HACCP in food businesses: the way ahead", Food Control, Vol. 6, No. 6, pp. 341-345, 1995.

[43] A. Eves y P. Dervisi, "Experiences of the implementation and operation of hazard analysis critical control points in the food service sector", International Journal of 
Hospitality Management, Vol. 24, No. 1, March, pp 3-19, 2005.

[44] S. Jin, J. Zhou and J. Ye, "Adoption of HACCP system in the Chinese food industry: A comparative analysis", Food Control, Vol. 19, No. 8, August, pp. 823-828, 2008.

[45] F. Untermann, "Food safety management and misinterpretation of HACCP", Food Control, Vol. 10, No. 3, June, pp. 161-167, 1999.

[46] T. Jackson, K. Harris and H. Cross, "International Meat and Poultry HACCP Alliance", Food Control, Vol. 7, No. 2, April, pp.103-105, 1996.

[47] Reglamento (CE) $n^{o} 852 / 2004$ del parlamento europeo y del consejo de 29 de abril de 2004, "Relativo a la higiene de los productos alimenticios", en Diario Oficial de la Unión Europea.

[48] N. Bell, M. Bell, T. Knowles, H. Whay, D. Main and A. Webster, "The development, implementation and testing of a lameness control programmed based on HACCP principles and designed for heifers on dairy farms", Veterinary Journal, Vol. 180, No. 2, May, pp. 178-188, 2009.

[49] C. Adams, "Hazard analysis and critical control point original "spin", Food Control, Vol. 13, pp. 355-358.

[50] S. Notermans and J. Jouve, "Quantitative risk analysis and HACCP: some remarks", Food Microbiology, Vol. 12, February, pp. 425-429, 1995 